\title{
Stochastic Simulator for Smart Microgrid Planning
}

\author{
Jesse Thornburg \\ Electrical \& Computer Engineering \\ Carnegie Mellon University, USA \\ jthornbu@andrew.cmu.edu
}

\author{
Bruce Krogh \\ Electrical \& Computer Engineering \\ Carnegie Mellon University, USA \\ krogh@rwanda.cmu.edu
}

\author{
Taha Selim Ustun \\ Electrical \& Computer Engineering \\ Carnegie Mellon University, USA \\ ustun@cmu.edu
}

\begin{abstract}
Developing world microgrids often balance insufficient supply with growing, unpredictable demand. Deterministic and probabilistic simulators exist to model these microgrids, and each focuses on different technical aspects. With the addition of smart meters into microgrids, monitoring and control is now available at high granularity, which enriches microgrid planning and operation. This research is designing a new simulator that incorporates models of smart meters that allow real-time power clipping for demand side management, effectively smoothing the system load curve as needed to represent inherent uncertainty. Individual supplies and demands are modeled as discrete probability mass functions (PMFs). To compare clipping schemes for grid operation and generation mixes for planning, we aggregate the probabilistic inputs by convolution and then compute expected energy sold and probability of avoiding power cuts. We compare these values for load profiles of different days of the week and test microgrid reliability with different numbers of customers clipped.
\end{abstract}

\section{Keywords}

smart grid; microgrid; power schedule; peak load reduction; DSM

\section{INTRODUCTION}

Microgrids consist of significantly different architectures and equipment mixes depending on a region's infrastructure and development level. Microgrids in the developing world constitute a special case, where customer demand is unpredictable and often grows rapidly when electricity becomes available for the first time. Another challenge in first-access microgrids comes when generation has limited capacity and is increasingly supplied by intermittent renewable sources [1].

Smart meters offer the monitoring and control needed to meet unpredictable loads with different mixes of supply resources. Smart meters on the market today have wired and wireless twoway communication and the ability to limit individual loads dynamically with power or current limits set remotely. Therefore smart grids need not rely on traditional hard-coded, binary control from simple current limiters. Smart meters allow automatic collection of data that is more frequent and more reliable, so by instrumenting each load and generator with a meter, a smart microgrid can empirically develop probabilistic models of each supply and demand node [2].

Permission to make digital or hard copies of part or all of this work for personal or classroom use is granted without fee provided that copies are not made or distributed for profit or commercial advantage and that copies bear this notice and the full citation on the first page. Copyrights for third-party components of this work must be honored. For all other uses, contact the Owner/Author.

Copyright is held by the owner/author(s) .

$A C M D E V^{\prime} 16$, November 17-22, 2016, Nairobi, Kenya

Copyright 2016 ACM 978-1-4503-4649-8/16/11.

http://dx.doi.org/10.1145/3001913.3006631
While tools exist for grid planning and some specifically for microgrid planning, many focus on topology and losses in the distribution grid (e.g., GridLAB-D) or on economic returns from the system (e.g., HOMER and the tool in [3]). Tools that are predominantly deterministic like HOMER have limited effectiveness for selecting and sizing generation technologies, since they take deterministic supply inputs that are fixed at each time step. For effectively planning a microgrid, probabilistic methods are needed to account for variation and uncertainty. Adding smart meters into a microgrid increases complexity by allowing loads to be individually monitored and controlled. Controllable demands present new variables to consider for planning and operation.

For modeling smart microgrids in the developing world, a new toolbox is needed for both planning and operation. The new simulator should account for supply intermittency and demand variation. It should allow for high growth in demand over time and load clipping when demand exceeds the available generation. To model this varying generator and consumer behavior, the simulator should treat each individually controlled node stochastically and allow for the demand at consumer nodes to be attenuated. This research is developing the features described above with the Load Attenuating Stochastic Simulator (LASS) [2].

Section 2 describes some complexities of microgrids and simulation tools that currently exist to address modeling difficulties. Section 3 explains the new functionalities that smart grids offer in a microgrid context. Section 4 gives an overview of LASS with an example of its capabilities from a case study based on a rural village microgrid in Rwanda.

\section{MICROGRIDS AND SIMULATION TOOLS}

Simulation tools for microgrids have grown with the grid technologies themselves. Since the early 1900's, graph tools like minimum spanning trees have helped lower the cost of distribution systems [4]. A more difficult problem arises when limited generation capacity is likely to be surpassed by system load, as occurs in rural microgrids of developing countries [1]. These problem scenarios become more likely for installations with intermittent renewable energy options like wind and solar.

Deterministic tools that are available at present look at systems of renewable distributed generation using precise computations of supply for each time step. But without a probabilistic accounting for input variations in these generation models, even a perfect control strategy will suffer when the assumptions for each input prove imprecise. Errors in the assumptions for each technology can compound when all the generation technologies are looked at in aggregate as a system's instantaneous generation mix.

The simulator in [3] uses its energy calculations to evaluate energy production and decide a control strategy. After these stages, the simulator predicts hourly energy flows and performs economic analysis by calculating cost per unit of electricity for each generator. Only after these stages does it perform a stochastic optimization step before recommending a final system configuration. The probabilistic results do not inform grid operation, though, so a different probabilistic simulator is needed for online, real-time control. 
Several probabilistic tools available today rely on Monte Carlo methods. While this approach can represent one or multiple contingencies across distributed generators, it generally cannot distinguish between probabilistic variations in behavior at the granularity of individually controlled loads [5]. For this granularity, an analytical method with PMFs representing each load and supply improves accuracy and precision when predicting system load and supply variations.

To plan for uncertainty and granularity, LASS is being developed as a stochastic simulator that does not rely on Monte Carlo methods. LASS accounts for variation by precisely calculating aggregate probabilities from PMF inputs. Each load and generator having their own PMF's - independent, discretized distributions - provides the granularity to accurately model independent agents in a microgrid system. To monitor and control the power consumption of these agents in real time, additional hardware must be added into the system beyond traditional load limiting devices [1]. Smart meters provide this functionality and are therefore a key part of microgrids modeled by LASS.

\section{SMART METERS FOR MICROGRIDS}

Smart meters enable monitoring of individual load consumption and generator production to high precision. Sampling rates can be set by the meter manufacturer or grid operator, and data collected can be stored locally and in the cloud. With these capabilities, smart meters provide the data to empirically create probabilistic models that account for the randomness inherent to a given system. As demonstrated in [1], smart meters are currently deployed in developing world microgrids as well as developed world grids. We view the microgrid as a network of nodes equipped with meters - each generator a supply node and each consumer a demand node. Though distribution lines are in place for power flow, data about power generation and consumption are transmitted wirelessly between the nodes and a central gateway processor (two-way communication). Demand nodes sample current and power consumed at frequencies of multiple $\mathrm{kHz}$, then the nodes' smart meters communicate the readings wirelessly to the gateway. Supply nodes each have a 3-phase meter that senses instantaneous generated capacity.

Depending on the total supply capacity available, the gateway manages each demand node and commands power or current limits that can depend on a specific customer's classification (high priority load vs. dispensable load, etc.). The demand nodes each receive their command (whether to clip and to what capacity) then carry out this command by allowing, limiting, or entirely cutting off their load's instantaneous consumption. In this way the gateway actively manages the demand loads with low latency. The gateway stores consumption data from all demand nodes and bills the customers accordingly. Customers can be charged different electricity prices, e.g., a tier of customers that agrees to have their load clipped at the grid manager's discretion would pay less per $\mathrm{kWh}$ than a tier that is never clipped.

\section{STOCHASTIC MICROGRID SIMULATOR}

LASS is a stochastic microgrid simulator built in MATLAB. LASS takes inputs for each supply and demand node, with each node modeled as a PMF at each time step. Using 1D convolution, it aggregates individual loads into one system load and similarly aggregates individual supplies into one system supply. A power capacity threshold is defined for each customer that has agreed to allow their demand to be clipped at times of low generation. With this threshold value, LASS defines a clipped demand PMF for each of these customers, and by $2 \mathrm{D}$ convolution the aggregate clipped demand PMFs are calculated to account for the effect of demand before clipping on the clipped result, i.e. the conditional probability is calculated given unclipped demand values. The resulting aggregate PMFs are then used to calculate expected energy sold and the probability of serving all customers without power cuts.

\subsection{PMF Inputs and Aggregation}

Demand inputs are classified by different payment tiers, where a given consumer's tier is decided by that consumer. Each tier is defined in LASS with a set electricity price and threshold, the maximum allowed power capacity when clipping is employed. The tier of consumers paying the highest electricity price receives the amount they demand without any clipping of their loads. Tier(s) paying lower electricity prices agree for their power to be clipped to an agreed-upon $T_{t}$ at all $t$ or during specific $t$ (e.g., peak demand hours in the evening or high-demand days).

For analysis with LASS, each consumer and generator in a microgrid is characterized by a PMF corresponding to the node power capacity at each time step $t$. All individual demand PMFs are aggregated into one PMF with $1 \mathrm{D}$ convolution. The aggregate demand PMF is referenced when total supply meets or exceeds total demand, i.e. when no reduction of load is needed. All combinations of tiers, clipped and unclipped, are similarly aggregated into PMFs corresponding to aggregated clipped demand, as shown in Figure 1. LASS then uses the aggregate supply PMF and all aggregate demand PMFs (unclipped and various levels of clipped) to calculate the probability of avoiding power cuts (quality of service) and expected energy sold by the suppliers at each $t$.

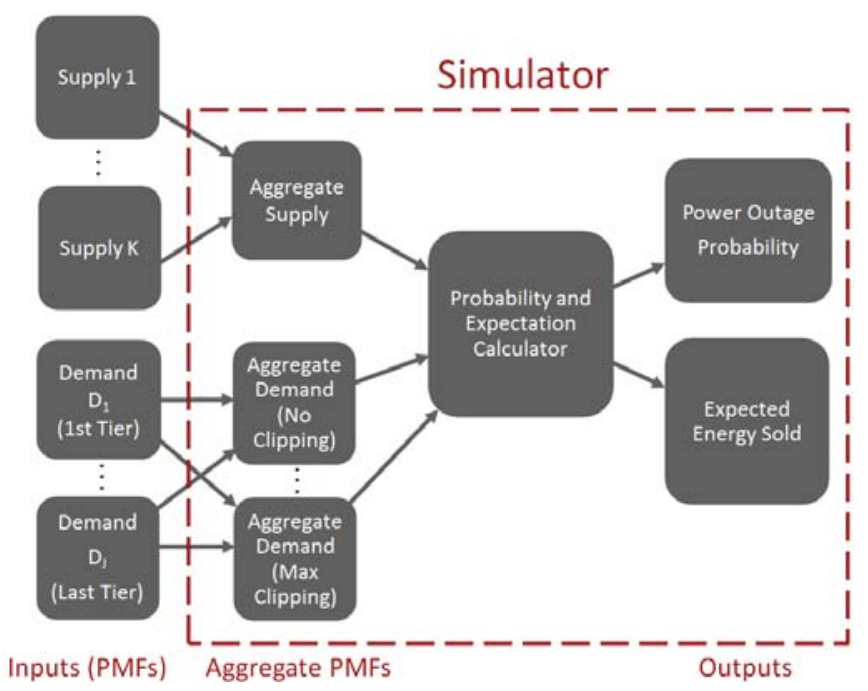

Figure 1. LASS Inputs and outputs

\subsection{Clipping Demand Nodes}

By clipping all loads in a tier to their threshold, the overall demand can be attenuated as needed if total capacity generated falls below total unclipped capacity demanded. Different numbers of customers are subjected to clipping depending on the limitations of the aggregate supply. As customers subject to clipping agree to their clipping threshold in exchange for less expensive electricity, the microgrid can meet its commitment to customers by providing less power to those in clipped tiers and using the power saved to supply demand nodes of higher paying or higher priority tiers (e.g., hospitals and security lights). In the following case study, demand and supply values were chosen to represent a microgrid that's chronically undersupplied (i.e. in need of demand clipping) on weekday evenings. 


\subsection{Case Study of Rwandan Village Microgrid}

The initial case study for LASS is based on a proposed microgrid for rural Rwanda presented in [2]. As listed in table 1, supply nodes include a solar PV array, a micro-hydro plant, and a diesel generator (total supply count $K=3$ ). The solar PV generation is modeled as ramping up to a maximum value depending on the hour of day and producing no power at night. At midday the $\mathrm{PV}$ array produces a maximum output of $3.5 \mathrm{~kW}$ in the absence of cloud cover. The micro-hydro plant is modeled as a binomial distribution about a mean value (the nominal or rated output of the plant) with $15 \mathrm{~kW}$ maximum output. The diesel generator is modeled as a $5 \mathrm{~kW}$ capacity input, modeled as constant given that generator failures are not being analyzed in this example. The aggregate supply is shown in Figure 2 [2].

Table 1. Summary of generation and loads in case study

\begin{tabular}{|l|l|}
\hline Supply & Demand \\
\hline$\cdot$ PV array $(\max 3.5 \mathrm{~kW})$ & $\bullet 1$ hospital $(\max 5 \mathrm{~kW})$ \\
$\bullet$ Micro hydro $(\max 15 \mathrm{~kW})$ & $\bullet 100$ homes $(\max 250 \mathrm{~W}$ each \\
- Diesel generator $(5 \mathrm{~kW})$ & without clipping, 50W clipped) \\
\hline
\end{tabular}

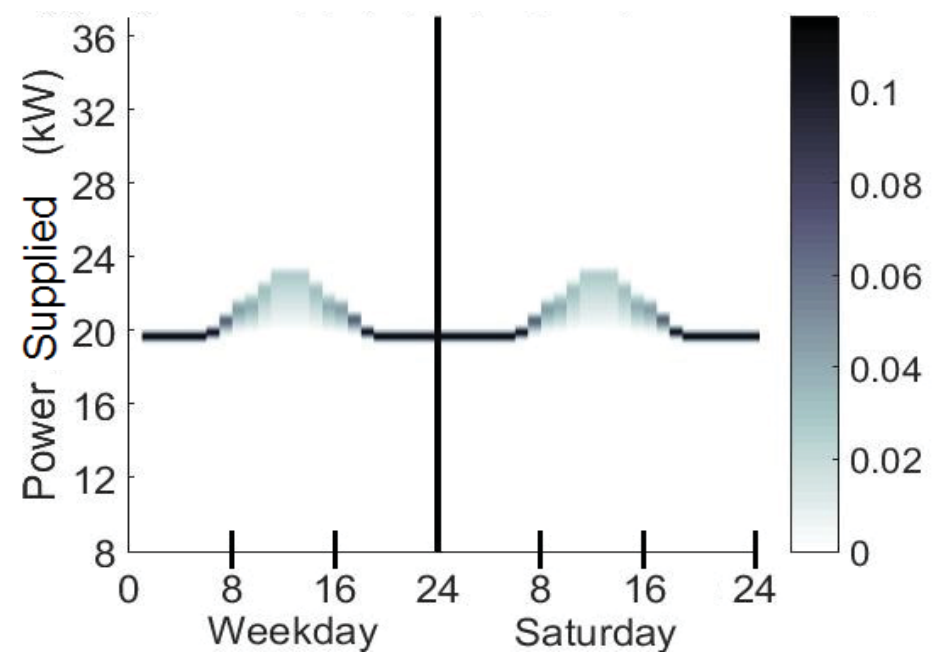

Figure 2. Aggregate supply (PV, hydro, and diesel) as PMFs

Demand nodes are a hospital and 100 households (see table 1). The hospital is deemed high priority and its demand is provided unclipped whenever possible. Hospital demand varies from 9 to $10 \mathrm{~kW}$ and is modeled as binomially distributed. Household demand is similarly binomially distributed based on published home load profiles [2]. Each household's demand varies from 0 to $250 \mathrm{~W}$ given the low consumption of lights and cell phone charging that constitute the majority of loads in a microgrid of rural Rwanda. The expected first-access home consumption of $250 \mathrm{~W}$ was chosen to allow slight demand increase from the upper limit available with solar home kits in Rwanda. German supplier Mobisol is used as a reference; their 200 Watt home system in Rwanda powers a TV, cell charger, and home lighting [2]. The case study households are split into clipped and unclipped tiers, the latter choosing to pay a higher rate per $\mathrm{kWh}$ for a higher level of service. Alternately, the unclipped households could represent nodes with traditional breakers but no smart meters. Clipped households are considered the lower tier and given last priority in this scenario, so they receive their full unclipped demand only when aggregate supply is high enough to supply all their demands plus that of the hospital and unclipped households. The aggregate demand PMFs are shown in Figure 3. Peak demand before clipping far exceeds peak supply (Figure 2) and occurs on weekday evenings when household consumption peaks.

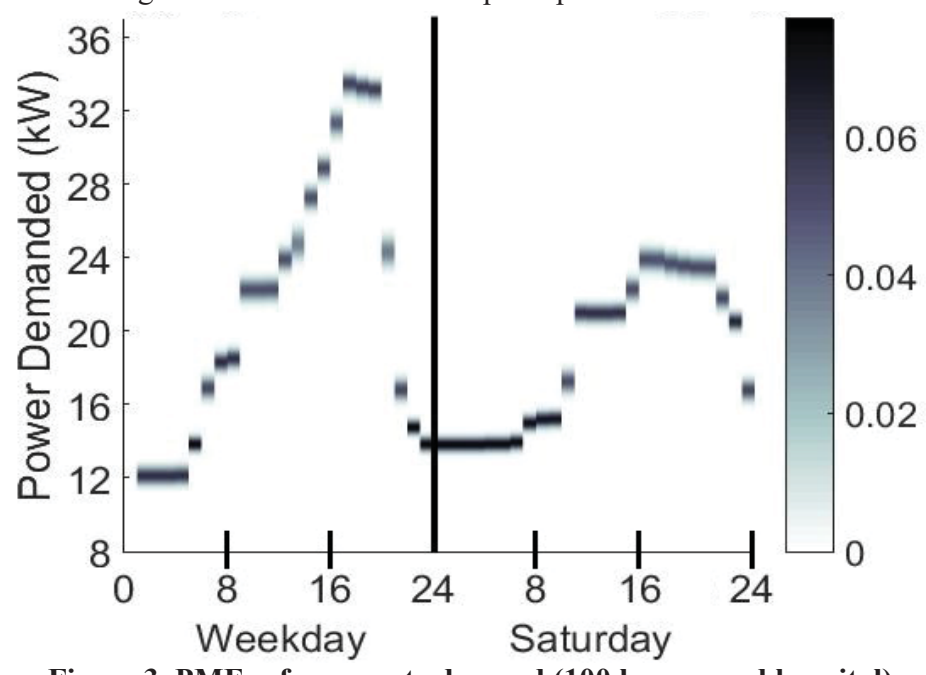

Figure 3. PMFs of aggregate demand (100 houses and hospital)

When supply is insufficient to meet all demand, lower tier households are clipped to a threshold capacity of $50 \mathrm{~W}$ until a future $t$ when aggregate supply is sufficient. The $50 \mathrm{~W}$ threshold was chosen to meet home lighting and cell phone charging needs in Rwanda, as described in [2]. The high tier (hospital and unclipped houses) has no power threshold since these loads are given priority to receive their full demand. If total supply cannot meet the reduced demand even after low tier loads have been cut, both tiers experience a power cut for that $t$. A well-equipped hospital would have its own backup generator for emergency scenarios, but because a node-specific backup supply like this would not feed into the rest of the microgrid we don't include such a generator in the case study. Each time step $t$ corresponds to one hour, and a typical week was analyzed with standard operation at all nodes - no emergency scenarios. Two days with different home load behavior are focused on in [2], a typical weekday and a typical weekend day (Saturday).

\subsection{Case Study Simulation Results}

As shown in Figure 4 and 5, certain high-demand hours (especially weekday evenings) are essentially certain to experience power cuts and deliver no energy in the absence of clipping [2]. At such times when demand exceeds supply, the level of clipping must be carefully chosen to sell nearly the full amount of electricity being generated. Figure 4 shows how expected energy sold grows with the number of customers clipped up to a certain tipping point (e.g., $75 \%=75$ homes for a weekday evening). Tipping points are time dependent and represent the percentage of customers clipped above which customers are being unnecessarily clipped and available power is not being sold to customers. Clipping fewer customers means risking power cuts when demand cannot be met.

Early in each day when demand is low, the aggregate supply is essentially certain to meet unclipped aggregate demand with no power cuts. Looking closer at the weekday plots (first 24 hours of figures 4 and 5), we see how power cuts become more likely as demand increases (figure 3 ) and supply decreases (figure 2), so that clipping is needed most in the late afternoon and evening. From figure 5 we see that from hours 1-8 all clipping levels including "no clipping" will result in no power cuts with probability 1. As the weekday demand increases from hours 9-17, more 


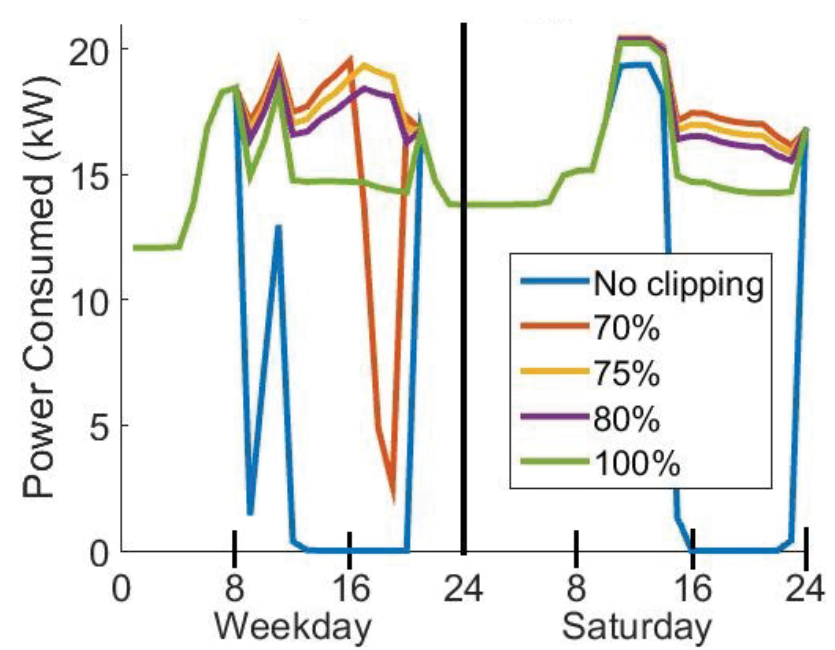

Figure 4. Expected energy sold with clipping varied

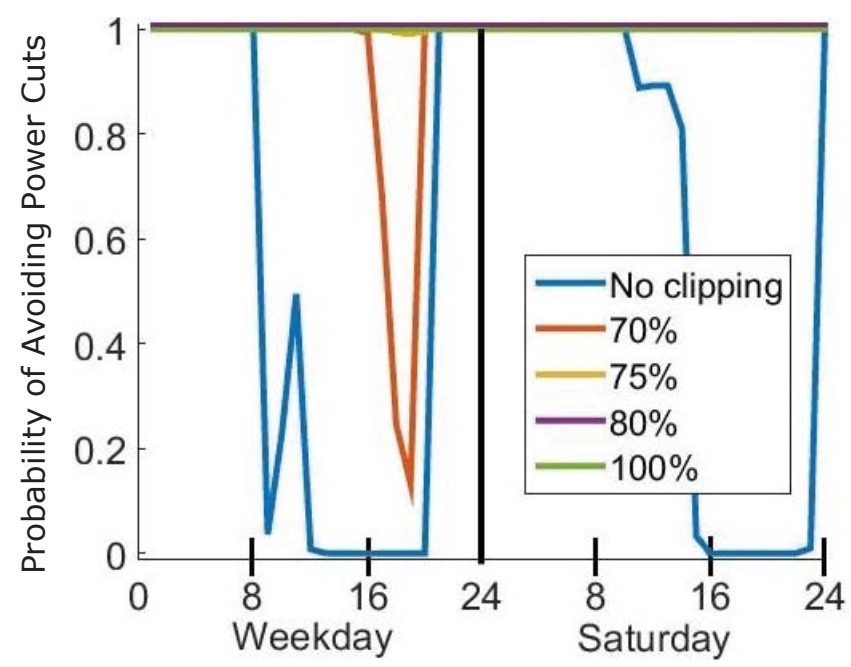

Figure 5. Probability of avoiding power cuts with clipping varied

clipping is needed to avoid power cuts, and only clipping at or above $75 \%$ of homes will ensure no power cuts with probability above 0.99 . Clipping at least $80 \%$ of homes ensures aggregate supply is sufficient but also results in less power sold to customers than at $75 \%$ clipping (see figure 4 ). As the demand decreases on a weekday evening less clipping is needed, and from hours 2124 there is again no need for clipping to prevent power cuts.

Because microgrid income increases with energy sold, the percentage of clipping employed at each $t$ affects both the probability of power cuts (the measure of quality of service used in [2]) and the gross income generated. In this way grid operation can be improved with a probabilistic simulator. Grid planning can also be improved by choosing generation size and type (e.g., base load plants like hydro and diesel generators) after seeing the expected value of aggregate demand (Figure 3 ). Note the cumulative results from figures 4 and 5 compiled in Table 2. The highlighted lines show that while clipping $75 \%$ of homes results in the max energy sold on a weekday, clipping fewer homes results in more energy sold on a Saturday, when typical home demand is lower. The optimal clipping level, therefore, is time and day dependent.

\section{CONCLUSIONS}

This paper presents an overview of microgrids in the developing world and the new functionalities introduced by smart meters.
Table 2. Cumulative results (highlighting max energy sold)

\begin{tabular}{|r|r|c|c|}
\cline { 2 - 3 } \multicolumn{1}{c|}{} & $\begin{array}{c}\text { Percentage of } \\
\text { homes clipped } \\
\text { (of 100 homes) }\end{array}$ & $\begin{array}{c}\text { Expected total } \\
\text { power cut duration } \\
\text { [hours/day] }\end{array}$ & $\begin{array}{c}\text { Expected } \\
\text { energy sold } \\
\text { [kWh/day] }\end{array}$ \\
\hline \multirow{4}{*}{ Weekday } & 0 (No clipping) & 11.2303 & 197.17 \\
\cline { 2 - 2 } & $70 \%$ & 1.9474 & 360.59 \\
\cline { 2 - 3 } & $75 \%$ & 0.0137 & 392.31 \\
\cline { 2 - 3 } & $80 \%$ & 0.0005 & 385.33 \\
\cline { 2 - 2 } Saturday & $100 \%$ & 0.0005 & 356.29 \\
\cline { 2 - 3 } & 0 (No clipping) & 9.4835 & 240.20 \\
\cline { 2 - 3 } & $70 \%$ & 0.0005 & 396.87 \\
\cline { 2 - 3 } & $75 \%$ & 0.0005 & 392.89 \\
\cline { 2 - 2 } & $80 \%$ & 0.0005 & 388.92 \\
\cline { 2 - 2 } & $100 \%$ & 0.0005 & 373.13 \\
\hline
\end{tabular}

The smart meter and microgrid system in [1] provide an illustrative example. Given the capabilities of a smart microgrid, a probabilistic simulator can be used to account for supply and demand variation in microgrid planning and operation. LASS is the tool proposed for this purpose in [2] which assumes no distribution line losses, i.e. all generator and supply nodes are assumed to be on a single bus. For microgrids where this lossless assumption could prove problematic, e.g., with substandard distribution lines or long distances between nodes, the system should be analyzed in parallel with both LASS and GridLAB-D, the latter for line loss calculations. LASS is currently run in the MATLAB command line, but a GUI is being developed to generate PMF inputs automatically based on parameters given by the user. Example choices for defining new PMFs would be distribution type (binomial, Poisson, bimodal, etc.) and corresponding parameters (maximum, minimum, mean, standard deviation) plus power increment for analysis, power threshold for clipping tiers, and percentage of customers in each clipping tier. LASS's capabilities are explored in a case study based on a Rwandan village. The case study finds room for economic and quality-of-service benefits if smart meters were operated to clip consumer loads to different extents in cases of limited and variable generation capacity. Both the expected energy sold and the probability of avoiding blackouts can be increased by using a probabilistic simulator to constantly monitor supply and demand while matching demand side management levels to a dynamic generation mix. LASS provides insights for both microgrid planning and operation.

\section{ACKNOWLEDGEMENTS}

This work was completed under NSF Grant No. DGE-1252522.

\section{REFERENCES}

[1] M. Buevich et al., "A system for fine-grained remote monitoring, control and pre-paid electrical service in rural microgrids," ACM/IEEE IPSN, 2014.

[2] J. Thornburg, T.S. Ustun, and B. Krogh, "Smart microgrid operation simulator for management and electrification planning," IEEE PES PowerAfrica, 2016.

[3] R. Carapellucci and L. Giordano, "Modeling and optimization of an energy generation island based on renewable technologies and hydrogen storage systems," International Journal of Hydrogen Energy, vol. 37, no. 3, pp. 2081-2093, 2012.

[4] R.L. Graham and P. Hell, "On the history of the minimum spanning tree," Annals of the History of Computing, vol. 7, no. 1, pp. 43-57, Jan 1985.

[5] B. Zhaohong et al., "Reliability evaluation of active distribution systems including microgrids," IEEE Transactions on Power Systems, vol. 27, no. 4, pp. 2342-2350, Nov. 2012. 